\title{
Specific genetic and microbial signature associated with paediatric ileal Crohn's disease
}

C ore gene expression profiles and microbial communities have been identified in the affected and unaffected ilea of patients with Crohn's disease, which were not present in patients with ulcerative colitis or control patients (without IBD). "We identified, for the first time, specific bacteria and genes that can be used to accurately classify the type of IBD that a patient has, and to predict the likelihood that they will go into remission with treatment," says Lee Denson, corresponding author.

IBD is thought to be caused by an inappropriate immune response to the gut microbiota in genetically susceptible hosts. "However, it was not known whether these microbial shifts and changes in host gene expression would be detected at diagnosis in the gut of children with Crohn's disease, or whether these would be associated with clinical outcomes," says Denson. Currently, only a small number of patients with IBD experience durable remission-there is a need to test whether genomic and microbial data could help improve diagnosis and treatment decisions.

The researchers focused on the ileum as it is likely to have a central role in the pathogenesis of Crohn's disease. "We decided to use state-of-the-art sequencing approaches and ileal biopsy samples to detect the widest range of microbial shifts and changes in host gene expression possible present in the actual lining of the gut," explains Denson. The ileal biopsy samples were obtained at the time of diagnosis from patients in the RISK cohort, a large treatment-naive prospective cohort of paediatric patients with IBD.

The first step in the study was identifying the core gene expression signature in ileal Crohn's disease. DUOX2 was noted within the top five most upregulated genes, and $A P O A 1$ was noted as the top downregulated gene. Furthermore, two gene coexpression signatures associated with these genes could then be defined.

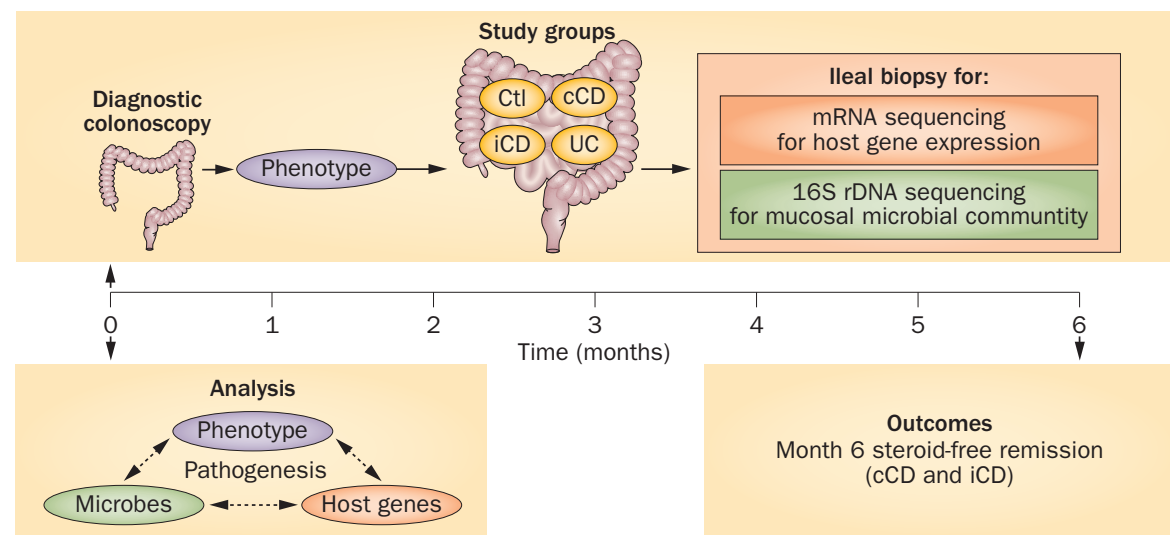

Image produced in consultation with J. Squires, L. Denson and Y. Haberman. Abbreviations: CCD, colon-only Crohn's disease; CtI, control; iCD, ileal Crohn's disease; UC, ulcerative colitis.

The researchers went on to investigate the gene expression profile of the unaffected ileum in patients with colon-only Crohn's disease. Importantly, they found that the $A P O A 1$ coexpression signature was enriched in the ilea of these patients, but not in patients with ulcerative colitismeaning that this signature is specific to Crohn's disease and independent of clinical inflammation. Differentiating colon-only Crohn's disease from ulcerative colitis can be tricky and Denson and colleagues believe that this genetic information could be used to classify patient groups.

The team then turned their attention to the microbial communities present in these paediatric patients with IBD. They performed a univariate analysis to investigate associations between the ileal microbiota and disease state in patients with Crohn's disease or ulcerative colitis, and non-IBD controls. As expected, an abundance of Firmicutes was observed in the ileum of non-IBD controls and patients with ulcerative colitis, and an expansion of Proteobacteria in the Crohn's disease ileum.

A central question at this stage was whether there was any difference in the ileal microbial community in patients with colon-only Crohn's disease and those with ileal involvement. Interestingly, a similar microbial profile was noted in both of these groups of patients, suggesting that this change in microbiota is a primary event that arises independently of gut inflammation.

To bring their findings together, the researchers then performed a multivariate analysis to test for associations between the gene coexpression signatures and the specific microbial profiles. An increase in DUOX2 expression was associated with an expansion of Proteobacteria across all forms of IBD, whereas downregulation of $A P O A 1$ expression in association with depletion of certain Firmicutes and Bacteroidetes taxa was found to be specific for Crohn's disease.

Finally, the influence of these genetic and microbial signatures on clinical outcome was assessed. A regression model that included genetic, microbial and clinical parameters was able to more accurately predict steroid-free remission at 6 months than a model that only included clinical factors. "The next step in this research will be to test whether targeting the microbial and host pathways that we have identified as associated with better outcomes in the patients will reduce inflammation in animal models," concludes Denson.

\section{Isobel Leake}

Original article Haberman, Y. et al. Pediatric Crohn disease patients exhibit specific ileal transcriptome and microbiome signature. J. Clin. Invest. doi:10.1172/JCI75436 